\title{
CERTAIN SUBCLASS OF ANALYTIC AND MULTIVALENT FUNCTIONS DEFINED BY USING A CERTAIN FRACTIONAL DERIVATIVE OPERATOR
}

\author{
M. K. AOUF AND B. A. FRASIN
}

\begin{abstract}
Making use of a certain operator of fractional derivative, a new subclass $F_{\lambda}(n, p, \alpha, \mu)$ of analytic and p-valent functions with negative coefficients is introduced and studied here rather systematically. Coefficient estimates, a distortion theorem and radii of p-valently closeto-convexity, starlikeness and convexity are given. Finally several applications involving an integral operator and a certain fractional calculus operator are also considered.
\end{abstract}

\section{INTRODUCTION}

Let $T_{p}(n)$ denote the class of functions of the form :

$$
f(z)=z^{p}-\sum_{k=n+p}^{\infty} a_{k} z^{k}\left(a_{k} \geq 0 ; p, n \in \mathbb{N}=\{1,2, \ldots\}\right),
$$

which are analytic and p-valent in the open unit disc $U=\{z:|z|<1\}$. Various operators of fractional calculus (that is, fractional integral and fractional derivative) have been studied in the literature rather extensively (cf., e.g., [9], [11], [12] and [13]; see also the various references cited therein). For our present investigations, we recall the following definitions.

Definition 1 (Fractional Integral Operator). The frcational integral operator of order $\lambda$ is defined, for a function $f(z)$ by

$$
D_{z}^{-\lambda} f(z)=\frac{1}{\Gamma(\lambda)} \int_{0}^{z} \frac{f(\zeta)}{(z-\zeta)^{1-\lambda}} d \zeta \quad(\lambda>0),
$$

where $f(z)$ is an analytic function in a simply-connected region of the $z$ plane containing the origin, and the multiplicity of $(z-\zeta)^{\lambda-1}$ is removed by requiring $\log (z-\zeta)$ to be real when $z-\zeta>0$.

2010 Mathematics Subject Classification. 30C45.

Key words and phrases. Multivalent functions, fractional calculus. 
Definition 2 (Fractional Derivative Operator). The fractional derivative of order $\lambda$ is defined, for a function $f(z)$ by

$$
D_{z}^{\lambda} f(z)=\frac{1}{\Gamma(1-\lambda)} \frac{d}{d z} \int_{0}^{z} \frac{f(\zeta)}{(z-\zeta)^{\lambda}} d \zeta \quad(0 \leq \lambda<1),
$$

where $f(z)$ is constrained, and the multiplicity of $(z-\zeta)^{-\lambda}$ is removed, as in Definition 2.

Definition 3 (Extended Fractional Derivative Operator). Under the hypothesis of Definition 3, the fractional derivative of order $n+\lambda$ is defined, for a function $f(z)$ by

$$
D_{z}^{n+\lambda} f(z)=\frac{d^{n}}{d z^{n}} D_{z}^{\lambda} f(z) \quad\left(0 \leq \lambda<1 ; n \in \mathbb{N}_{0}=\mathbb{N} \cup\{0\}\right) .
$$

In terms of the fractional derivative operator $D_{z}^{\lambda}$ of order $\lambda$, defined by (1.3), with

$$
D_{z}^{0} f(z)=f(z) \text { and } D_{z}^{1} f(z)=f^{\prime}(z)
$$

Srivastava and Aouf [11] defined and studied the operator:

$$
\Omega_{z}^{(\lambda, p)} f(z)=\frac{\Gamma(p+1-\lambda)}{\Gamma(p+1)} z^{\lambda-p} D_{z}^{\lambda} f(z) \quad(0 \leq \lambda \leq 1 ; p \in \mathbb{N}) .
$$

In this paper we shall study some properties of the class $F_{\lambda}(n, p, \alpha, \mu)$, defined as follows:

Definition 4. Let $F_{\lambda}(n, p, \alpha, \mu)$ be the subclass of $T_{p}(n)$ consisting of functions of the form (1.1) which satisfy the following inequality:

$$
\Re\left\{\frac{\Gamma(p+1-\lambda)}{\Gamma(p+1)} z^{\lambda-p}\left[(1-\mu) D_{z}^{\lambda} f(z)+\frac{\mu}{p} z\left(D_{z}^{\lambda} f(z)\right)^{\prime}\right]\right\}>\frac{\alpha}{p}
$$

where $z \in U ; 0 \leq \alpha<p ; p \in \mathbb{N} ; 0 \leq \lambda \leq 1 ; \alpha+\lambda<p ; 0 \leq \mu \leq 1)$.

By specializing the parameters $\lambda, \mu, \alpha$ and $p$, we obtain the following subclasses studied by various authors:

(i) $F_{\lambda}(n, 1, \alpha, \mu)=F_{\lambda}(n, \alpha, \mu) \quad(0 \leq \alpha<1)$ (Altintas et al. [1]);

(ii) $F_{0}(1, p, \alpha, \mu)=F_{p}(\mu, \alpha) \quad(0 \leq \alpha<p ; p \in \mathbb{N} ; \mu \geq 0)$ (Lee et al. [6] and Aouf and Darwish [2]);

(iii) $F_{0}(1,1, \alpha, \mu)=F_{\mu}(\alpha) \quad(0 \leq \alpha<1 ; 0 \leq \mu \leq 1)$ (Bhoosnurmath and Swamy [4]);

$$
\begin{aligned}
& \text { (iv) } F_{\lambda}(1, p, p \alpha, 0)=F_{p}(\alpha, \lambda) \quad(0 \leq \alpha<1 ; 0 \leq \lambda \leq 1 ; p \in \mathbb{N}) \\
& =\left\{f(z) \in T_{p}(1)=T_{p}: \Re\left[\frac{\Gamma(p+1-\lambda)}{\Gamma(p+1)} z^{\lambda-p} D_{z}^{\lambda} f(z)\right]>\alpha, z \in U\right\}
\end{aligned}
$$


(see $[10])$;

(v) $F_{0}(1, p, p \alpha, 0)=F_{p}(0, p \alpha) \quad(0 \leq \alpha<1)$

$$
=\left\{f(z) \in T_{p}(1)=T_{p}: \Re\left(\frac{f(z)}{z^{p}}\right)>\alpha, \quad z \in U\right\} ;
$$

(see $[6])$

(vi) $F_{0}(1, p, \alpha, 1)=F_{p}(1, \alpha) \quad(0 \leq \alpha<p)$

$$
=\left\{f(z) \in T_{p}(1)=T_{p}: \Re\left(\frac{f^{\prime}(z)}{z^{p-1}}\right)>\alpha, z \in U\right\} .
$$

$($ see $[6])$.

Also we note that: $F_{\lambda}(n, p, \alpha, 1)=F_{\lambda}(n, p, \alpha)$

$$
=\left\{f(z) \in T_{p}(n): \Re\left[\frac{\Gamma(p+1-\lambda)}{\Gamma(p+1)} \cdot \frac{\left(D_{z}^{\lambda} f(z)\right)^{\prime}}{z^{p-\lambda-1}}\right]>\alpha\right\},
$$

where $0 \leq \alpha<p, p \in \mathbb{N}, \quad z \in U$.

In our present paper, we shall make use of the familiar operator $J_{c, p}$ defined by (cf. [3], [7] and [8]; see also [12])

$$
\left(J_{c, p} f\right)(z)=\frac{c+p}{z^{c}} \int_{0}^{z} t^{c-1} f(t) d t(c>-p) .
$$

\section{Coefficient estimates}

Theorem 1. Let the function $f(z) \in T_{p}(n)$ be given by (1.1). Then $f(z) \in$ $F_{\lambda}(n, p, \alpha, \mu)$ if and only if

$$
\sum_{k=n+p}^{\infty} \frac{[p+\mu(k-p-\lambda)] \Gamma(k+1) \Gamma(p+1-\lambda)}{\Gamma(p+1) \Gamma(k+1-\lambda)} a_{k} \leq p-\mu \lambda-\alpha
$$

where $\alpha+\lambda<p$.

Proof. Assume that the inequality (2.1) holds true. Then we find that

$$
\begin{aligned}
& \left|\frac{\Gamma(p+1-\lambda)}{\Gamma(p+1)} z^{\lambda-p}\left[(1-\mu) D_{z}^{\lambda} f(z)+\frac{\mu}{p} z\left(D_{z}^{\lambda} f(z)\right)^{\prime}\right]-\left(1-\frac{\mu}{p} \lambda\right)\right| \\
& =\left|-\sum_{k=n+p}^{\infty}\left[\frac{p+\mu(k-p-\lambda)}{p} \frac{\Gamma(k+1) \Gamma(p+1-\lambda)}{\Gamma(p+1) \Gamma(k+1-\lambda)}\right] a_{k} z^{k-p}\right| \\
& \leq \sum_{k=n+p}^{\infty} \frac{[p+\mu(k-p-\lambda)] \Gamma(k+1) \Gamma(p+1-\lambda)}{\Gamma(p+1) \Gamma(k+1-\lambda)} a_{k} \leq p-\mu \lambda-\alpha,
\end{aligned}
$$

where $z \in U ; 0 \leq \alpha<p ; \alpha+\lambda<p ; p, n \in \mathbb{N} ; 0 \leq \lambda \leq 1 ; 0 \leq \mu \leq 1$. 
This shows that the values of the function

$$
\Phi(z)=\frac{\Gamma(p+1-\lambda)}{\Gamma(p+1)} z^{\lambda-p}\left[(1-\mu) D_{z}^{\lambda} f(z)+\frac{\mu}{p} z\left(D_{z}^{\lambda} f(z)\right)^{\prime}\right]
$$

lie in a circle which is centered at $w=1-\frac{\mu \lambda}{p}$ and whose radius is $1-\frac{\mu \lambda}{p}-\alpha$.

Hence $f(z)$ satisfies the condition (1.7).

Conversely, assume that the function $f(z)$ defined by (1.1) is in the class $F_{\lambda}(n, p, \mu, \alpha)$. Then we have

$$
\Re\left\{1-\frac{\mu}{p} \lambda-\sum_{k=n+p}^{\infty}\left[\frac{p+\mu(k-p-\lambda)}{p}\right] \frac{\Gamma(k+1) \Gamma(p+1-\lambda)}{\Gamma(p+1) \Gamma(k+1-\lambda)} a_{k} z^{k-p}\right\}>\frac{\alpha}{p}
$$

for some $\alpha(0 \leq \alpha<p), 0 \leq \lambda \leq 1, \alpha+\lambda<p, 0 \leq \mu \leq 1, p, n \in \mathbb{N}$ and $z \in U$. Choose values of $z$ on the real axis so that $\Phi(z)$ given by $(2.2)$ is real.

Letting $z \rightarrow 1^{-}$through real values, we can see that

$$
p-\mu \lambda-\sum_{k=n+p}^{\infty} \frac{[p+\mu(k-p-\lambda)] \Gamma(k+1) \Gamma(p+1-\lambda)}{\Gamma(p+1) \Gamma(k+1-\lambda)} a_{k} \geq \alpha
$$

for $0 \leq \alpha<p ; p, n \in \mathbb{N} ; 0 \leq \lambda \leq 1 ; \alpha+\lambda<p ; 0 \leq \mu \leq 1$, which is equivalent to the assertion (2.1) of Theorem 1 .

Putting $p=1$ in Theorem 1, we obtain the following result.

Corollary 1. Let the function $f(z)$ be defined by (1.1) (with $p=1$ ). Then $f(z) \in F_{\lambda}(n, \alpha, \mu)$ if and only if

$$
\sum_{k=n+1}^{\infty} \frac{[1+\mu(k-1-\lambda)] \Gamma(k+1) \Gamma(2-\lambda)}{\Gamma(k+1-\lambda)} a_{k} \leq 1-\mu \lambda-\alpha
$$

for $\alpha+\lambda<1$.

Remark 1. We note that the result obtained by Altintas et al. [1, Theorem $1]$ is not correct. The correct result is given by Corollary 1.

Corollary 2. Let the function $f(z)$ be defined by (1.1) be in the class $F_{\lambda}(n, p, \alpha, \mu)$. Then

$$
a_{k} \leq \frac{(p-\mu \lambda-\alpha) \Gamma(p+1) \Gamma(k+1-\lambda)}{[p+\mu(k-p-\lambda)] \Gamma(k+1) \Gamma(p+1-\lambda)}
$$

for $k \geq n+p ; p, n \in \mathbb{N}$.

The result is sharp for the function $f(z)$ given by

$$
f(z)=z^{p}-\frac{(p-\mu \lambda-\alpha) \Gamma(p+1) \Gamma(k+1-\lambda)}{[p+\mu(k-p-\lambda)] \Gamma(k+1) \Gamma(p+1-\lambda)} z^{k},
$$


where $k \geq n+p ; p, n \in \mathbb{N}$.

\section{Distortion theorem}

Theorem 2. If a function $f(z)$ defined by (1.1) is in the class $F_{\lambda}(n, p, \alpha, \mu)$, then

$$
\begin{aligned}
& \left\{\frac{p !}{(p-j) !}\right. \\
& \left.\quad-\frac{(p-\mu \lambda-\alpha) \Gamma(p+1) \Gamma(n+p+1-\lambda)(n+p) !}{[p+\mu(n-\lambda)] \Gamma(n+p+1) \Gamma(p+1-\lambda)(n+p-j) !}|z|^{n}\right\}|z|^{p-j} \\
& \leq\left|f^{(j)}(z)\right| \leq\left\{\frac{p !}{(p-j) !}\right. \\
& \left.+\frac{(p-\mu \lambda-\alpha) \Gamma(p+1) \Gamma(n+p+1-\lambda)(n+p) !}{[p+\mu(n-\lambda)] \Gamma(n+p+1) \Gamma(p+1-\lambda)(n+p-j) !}|z|^{n}\right\}|z|^{p-j},
\end{aligned}
$$

where $z \in U ; 0 \leq \alpha<p ; 0 \leq \lambda \leq 1 ; \alpha+\lambda<p ; p, n \in \mathbb{N} ; 0 \leq \mu \leq 1 ; j \in$ $\mathbb{N}_{0} ; p>j$.

The result is sharp for the function $f(z)$ given by

$$
f(z)=z^{p}-\frac{(p-\mu \lambda-\alpha) \Gamma(p+1) \Gamma(n+p+1-\lambda)}{[p+\mu(n-\lambda)] \Gamma(n+p+1) \Gamma(p+1-\lambda)} z^{n+p} \quad(p, n \in \mathbb{N}) .
$$

Proof. In view of Theorem 1, we have

$$
\begin{aligned}
& \frac{[p+\mu(n-\lambda)] \Gamma(n+p+1) \Gamma(p+1-\lambda)}{(p-\mu \lambda-\alpha) \Gamma(p+1) \Gamma(n+p+1-\lambda)(n+p) !} \sum_{k=n+p}^{\infty} k ! a_{k} \\
& \leq \sum_{k=n+p}^{\infty} \frac{[p+\mu(k-p-\lambda)] \Gamma(k+1) \Gamma(p+1-\lambda)}{(p-\mu \lambda-\alpha) \Gamma(p+1) \Gamma(k+1-\lambda)} a_{k} \leq 1
\end{aligned}
$$

which readily yields

$$
\sum_{k=j+p}^{\infty} k ! a_{k} \leq \frac{(p-\mu \lambda-\alpha) \Gamma(p+1) \Gamma(n+p+1-\lambda)(n+p) !}{[p+\mu(n-\lambda)] \Gamma(n+p+1) \Gamma(p+1-\lambda)} .
$$

Now, by differentiating both sides of (1.1) $j$ times, we obtain

$$
f^{(j)}(z)=\frac{p !}{(p-j) !} z^{p-j}-\sum_{k=n+p}^{\infty} \frac{k !}{(k-j) !} a_{k} z^{k-j},
$$

where $k \geq n+p ; p, n \in \mathbb{N} ; j \in \mathbb{N}_{0} ; p>j$.

Theorem 2 follows readily from (3.3) and (3.4). 
Finally, it is easy to see that the bounds in (3.1) are attained for the function $f(z)$ given by (3.2).

Putting (i) $p=1$ and $\lambda=j=0$ (ii) $p=j=1$ and $\lambda=0$ in Theorem 2, we obtain the following results.

Corollary 3. If a function $f(z)$ defined by (1.1) (with $p=1$ ) is in the class $F_{0}(n, 1, \alpha, \mu)=F_{0}(n, \alpha, \mu)$, then

$$
|z|-\frac{(1-\alpha)}{(1+\mu n)}|z|^{n+1} \leq|f(z)| \leq|z|+\frac{(1-\alpha)}{(1+\mu n)}|z|^{n+1} \quad(n \in \mathbb{N} ; z \in U) .
$$

The result is sharp.

Corollary 4. If a function $f(z)$ is defined by (1.1) (with $p=1$ ) is in the class $F_{0}(n, 1, \alpha, \mu)=F_{0}(n, \alpha, \mu)$, then

$1-\frac{(1-\alpha)(n+1)}{(1+\mu n)}|z|^{n} \leq\left|f^{\prime}(z)\right| \leq 1+\frac{(1-\alpha)(n+1)}{(1+\mu n)}|z|^{n} \quad(n \in \mathbb{N} ; z \in U)$.

The result is sharp.

Remark 2. We note that the results obtained by Altintas et al. [1, Corollary 8 and Corollary 9] are not correct. The correct results are given by (3.5) and (3.6), respectively;

Putting (i) $n=1$ and $\lambda=j=0$ (ii) $n=j=1$ and $\lambda=0$ in Theorem 2, we obtain the result obtained by Lee at al. [6, Theorem 3].

\section{RAdi of CLOSE-TO-CONVEXITY, STARLIKENESS AND CONVEXITY}

Theorem 3. Let the function $f(z)$ defined by (1.1) be in the class $F_{\lambda}(n, p$, $\alpha, \mu)$, then

(i) $f(z)$ is p-valently close-to-convex of order $\varphi(0 \leq \varphi<p)$ in $|z|<r_{1}$, where

$$
r_{1}=\inf _{k}\left\{\frac{[p+\mu(k-p-\lambda)] \Gamma(k) \Gamma(p+1-\lambda)(p-\varphi)}{(p-\mu \lambda-\alpha) \Gamma(p+1) \Gamma(k+1-\lambda)}\right\}^{\frac{1}{k-p}}
$$

for $k \geq n+p ; p, n \in \mathbb{N}$,

(ii) $f(z)$ is $p$-valently starlike of order $\varphi(0 \leq \varphi<p)$ in $|z|<r_{2}$, where

$$
r_{2}=\inf _{k}\left\{\frac{[p+\mu(k-p-\lambda)] \Gamma(k+1) \Gamma(p+1-\lambda)}{(p-\mu \lambda-\alpha) \Gamma(p+1) \Gamma(k+1-\lambda)}\left(\frac{p-\varphi}{k-\varphi}\right)\right\}^{\frac{1}{k-p}}
$$

for $k \geq n+p ; p, n \in \mathbb{N}$,

(iii) $f(z)$ is p-valently convex of order $\varphi(0 \leq \varphi<p)$ in $|z|<r_{3}$, where

$$
r_{3}=\inf _{k}\left\{\frac{[p+\mu(k-p-\lambda)] \Gamma(k) \Gamma(p+1-\lambda)(p-\varphi)}{(p-\mu \lambda-\alpha) \Gamma(p+1) \Gamma(k+1-\lambda)}\left(\frac{p-\varphi}{k-\varphi}\right)\right\}^{\frac{1}{k-p}}
$$


for $k \geq n+p ; p, n \in \mathbb{N}$. Each of these results is sharp for the function $f(z)$ given by (2.7).

Proof. (i) It is sufficient to show that

$$
\begin{aligned}
& \left|\frac{f^{\prime}(z)}{z^{p-1}}-p\right| \leq p-\varphi \quad\left(|z|<r_{1} ; 0 \leq \varphi<p ; p \in \mathbb{N}\right), \\
& \left|\frac{z f^{\prime}(z)}{f(z)}-p\right| \leq p-\varphi \quad\left(|z|<r_{2} ; 0 \leq \varphi<p ; p \in \mathbb{N}\right),
\end{aligned}
$$

and that

$$
\left|1+\frac{z f^{\prime \prime}(z)}{f^{\prime}(z)}-p\right| \leq p-\varphi \quad\left(|z|<r_{3} ; 0 \leq \varphi<p ; p \in \mathbb{N}\right)
$$

for a function $f(z) \in F_{\lambda}(n, p, \alpha, \mu)$, where $r_{1}, r_{2}$ and $r_{3}$ are defined by (4.1), (4.2) and (4.3), respectively.

Remark 3. (i) We note that the results obtained by Altintas et al. [1, Theorems 6 and 7 and Corollary 1] are not correct. The correct results are given by (4.1), (4.2) and (4.3) (with $p=1$ ), respectively;

(ii) Putting $n=1$ and $\lambda=0$ in Theorem 3, we obtain the results obtained by Aouf and Darwish [2, Theorems 6 and 7, Corollary 2, respectively].

\section{Applications of fractional CAlculus}

In this section, we shall investigate the growth and distortion properties of the operators $J_{c, p}$ and $D_{z}^{\lambda}$. In order to derive our results, we need the following lemma given by Chen et al. [5].

Lemma 1. (see [5], Chen et al.) Let the function $f(z)$ defined by (1.1). Then

$$
D_{z}^{\lambda}\left\{\left(J_{c, p} f\right)(z)\right\}=\frac{\Gamma(p+1)}{\Gamma(p+1-\lambda)} z^{p-\lambda}-\sum_{k=n+p}^{\infty} \frac{(c+p) \Gamma(k+1)}{(c+k) \Gamma(k+1-\lambda)} a_{k} z^{k-\lambda}
$$

where $\lambda \in R ; c>-p ; p, n \in \mathbb{N}$ and

$$
\begin{aligned}
J_{c, p}\left(D_{z}^{\lambda}\{f(z)\}=\frac{(c+p) \Gamma(p+1)}{(c+p-\lambda) \Gamma(p+1-\lambda)} z^{p-\lambda}\right. & \\
& -\sum_{k=n+p}^{\infty} \frac{(c+p) \Gamma(k+1)}{(c+k-\lambda) \Gamma(k+1-\lambda)} a_{k} z^{k-\lambda},
\end{aligned}
$$

where $\lambda \in R ; c>-p ; p, n \in \mathbb{N}$, provided that no zeros appear in the denominators in (5.1) and (5.2). 
Theorem 4. Let the function $f(z)$ defined by (1.1) be in the class $F_{\lambda}(n, p$, $\alpha, \mu)$. Then

$$
\begin{aligned}
& \left|D_{z}^{-\delta}\left\{\left(J_{c, p} f\right)(z)\right\}\right| \geq\left\{\frac{\Gamma(p+1)}{\Gamma(p+1+\delta)}\right. \\
& \left.\quad-\frac{(c+p)(p-\mu \lambda-\alpha) \Gamma(p+1) \Gamma(n+p+1-\lambda)}{(c+n+p)[p+\mu(n-\lambda)] \Gamma(n+p+1+\delta) \Gamma(p+1-\lambda)}|z|^{n}\right\}|z|^{p+\delta}
\end{aligned}
$$

for $z \in U ; 0 \leq \alpha<p ; 0 \leq \lambda \leq 1 ; \alpha+\lambda<p ; p, n \in \mathbb{N} ; 0 \leq \mu \leq 1 ; c>-p$ and

$$
\begin{aligned}
& \left|D_{z}^{-\delta}\left\{\left(J_{c, p} f\right)(z)\right\}\right| \leq\left\{\frac{\Gamma(p+1)}{\Gamma(p+1+\delta)}\right. \\
& \left.\quad+\frac{(c+p)(p-\mu \lambda-\alpha) \Gamma(p+1) \Gamma(n+p+1-\lambda)}{(c+n+p)[p+\mu(n-\lambda)] \Gamma(n+p+1+\delta) \Gamma(p+1-\lambda)}|z|^{n}\right\}|z|^{p+\delta}
\end{aligned}
$$

for $z \in U ; 0 \leq \alpha<p ; 0 \leq \lambda \leq 1 ; \alpha+\lambda<p ; p, n \in \mathbb{N} ; 0 \leq \mu \leq 1 ; c>-p$.

Each of the assertions (5.3) and (5.4) is sharp.

Proof. In view of Theorem 1, we have

$$
\begin{aligned}
& \frac{[p+\mu(n-\lambda)] \Gamma(n+p+1) \Gamma(p+1-\lambda)}{(p-\mu \lambda-\alpha) \Gamma(p+1) \Gamma(n+p+1-\lambda)} \sum_{k=n+p}^{\infty} a_{k} \\
& \leq \sum_{k=n+p}^{\infty} \frac{[p+\mu(k-p-\lambda)] \Gamma(k+1) \Gamma(p+1-\lambda)}{(p-\mu \lambda-\alpha) \Gamma(p+1) \Gamma(k+1-\lambda)} a_{k} \leq 1
\end{aligned}
$$

which readily yields

$$
\sum_{k=n+p}^{\infty} a_{k} \leq \frac{(p-\mu \lambda-\alpha) \Gamma(p+1) \Gamma(n+p+1-\lambda)}{[p+\mu(n-\lambda)] \Gamma(n+p+1) \Gamma(p+1-\lambda)} .
$$

Consider the function $F(z)$ defined in $U$ by

$$
\begin{aligned}
F(z) & =\frac{\Gamma(p+1+\delta)}{\Gamma(p+1)} z^{-\delta} D_{z}^{-\delta}\left\{\left(J_{c, p} f\right)(z)\right\} \\
& =z^{p}-\sum_{k=n+p}^{\infty} \frac{(c+p) \Gamma(k+1) \Gamma(p+1+\delta)}{(c+k) \Gamma(k+1+\delta) \Gamma(p+1)} a_{k} z^{k} \\
& =z^{p}-\sum_{k=n+p}^{\infty} \Phi(k) a_{k} z^{k} \quad(z \in U)
\end{aligned}
$$


where

$$
\Phi(k)=\frac{(c+p) \Gamma(k+1) \Gamma(p+1+\delta)}{(c+k) \Gamma(k+1+\delta) \Gamma(p+1)}
$$

for $k \geq n+p ; p, n \in \mathbb{N} ; \delta>0$. Since $\Phi(k)$ is a decreasing function of $k$ when $\delta>0$, we get

$$
0<\Phi(k) \leq \Phi(n+p)=\frac{(c+p) \Gamma(n+p+1) \Gamma(p+1+\delta)}{(c+n+p) \Gamma(n+p+1+\delta) \Gamma(p+1)}
$$

for $c>-p ; p, n \in \mathbb{N} ; \delta>0$

Thus, by using (5.6) and (5.8), we deduce that

$$
\begin{aligned}
& |F(z)| \geq|z|^{p}-\Phi(n+p)|z|^{n+p} \sum_{k=n+p}^{\infty} a_{k} \geq|z|^{p} \\
- & \frac{(c+p)(p-\mu \lambda-\alpha) \Gamma(p+1+\delta) \Gamma(n+p+1-\lambda)}{(c+n+p)[p+\mu(n-\lambda)] \Gamma(n+p+1+\delta) \Gamma(p+1-\lambda)}|z|^{n+p} \quad(z \in U),
\end{aligned}
$$

and

$$
\begin{aligned}
& |F(z)| \leq|z|^{p}+\Phi(n+p)|z|^{n+p} \sum_{k=n+p}^{\infty} a_{k} \leq|z|^{p} \\
+ & \frac{(c+p)(p-\mu \lambda-\alpha) \Gamma(p+1+\delta) \Gamma(n+p+1-\lambda)}{(c+n+p)[p+\mu(n-\lambda)] \Gamma(n+p+1+\delta) \Gamma(p+1-\lambda)}|z|^{n+p} \quad(z \in U),
\end{aligned}
$$

which yield the inequalities (5.3) and (5.4) of Theorem 4. The equalities in $(5.3)$ and (5.4) are attained for the function $f(z)$ given by

$$
\begin{aligned}
& D_{z}^{-\delta}\left\{\left(J_{c, p} f\right)(z)\right\}=\left\{\frac{\Gamma(p+1)}{\Gamma(p+1+\delta)}\right. \\
& \left.-\frac{(c+p)(p-\mu \lambda-\alpha) \Gamma(p+1+\delta) \Gamma(n+p+1-\lambda)}{(c+n+p)[p+\mu(n-\lambda)] \Gamma(n+p+1+\delta) \Gamma(p+1-\lambda)} z^{n}\right\} z^{p+\delta}
\end{aligned}
$$

or, equivalently, by

$$
\left(J_{c, p} f\right)(z)=z^{p}-\frac{(c+p)(p-\mu \lambda-\alpha) \Gamma(p+1) \Gamma(n+p+1-\lambda)}{(c+n+p)[p+\mu(n-\lambda)] \Gamma(n+p+1) \Gamma(p+1-\lambda)} z^{n+p} .
$$

Thus we complete the proof of Theorem 4 .

Using arguments similar to those in the proof of Theorem 4, we obtain the following result. 
Theorem 5. Let the function $f(z)$ defined by (1.1) be in the class $F_{\lambda}(n, p$, $\alpha, \mu)$. Then

$$
\begin{aligned}
& \left|D_{z}^{\delta}\left\{\left(J_{c, p} f\right)(z)\right\}\right| \geq\left\{\frac{\Gamma(p+1)}{\Gamma(p+1-\delta)}\right. \\
& \left.\quad-\frac{(c+p)(p-\mu \lambda-\alpha) \Gamma(p+1) \Gamma(n+p+1-\lambda)}{(c+n+p)[p+\mu(n-\lambda)] \Gamma(n+p+1-\delta) \Gamma(p+1-\lambda)}|z|^{n}\right\}|z|^{p-\delta},
\end{aligned}
$$

where $z \in U ; 0 \leq \alpha<p ; 0 \leq \lambda \leq 1 ; \alpha+\lambda<p ; p, n \in \mathbb{N} ; 0 \leq \mu \leq 1 ; c>-p$ and

$$
\begin{aligned}
& \left|D_{z}^{\delta}\left\{\left(J_{c, p} f\right)(z)\right\}\right| \leq\left\{\frac{\Gamma(p+1)}{\Gamma(p+1-\delta)}\right. \\
& \left.\quad+\frac{(c+p)(p-\mu \lambda-\alpha) \Gamma(p+1) \Gamma(n+p+1-\lambda)}{(c+n+p)[p+\mu(n-\lambda)] \Gamma(n+p+1-\delta) \Gamma(p+1-\lambda)}|z|^{n}\right\}|z|^{p-\delta},
\end{aligned}
$$

where $z \in U ; 0 \leq \alpha<p ; 0 \leq \lambda \leq 1 ; \alpha+\lambda<p ; p, n \in \mathbb{N} ; 0 \leq \mu \leq 1 ; c>$ $-p$. Each of the assertion (5.11) and (5.12) is sharp.

Remark 4. (i) Putting $\lambda=0$ and $n=1$ in Theorems 4 and 5, we obtain the corresponding results for the class $F_{0}(p, \alpha, \mu)$;

(ii) Putting $p=1$ in Theorems 4 and 5, we obtain the corresponding results for the class $F_{\lambda}(n, \alpha, \mu)$.

Theorem 6. Let the function $f(z)$ defined by (1.1) be in the class $F_{\lambda}(n, p$, $\alpha, \mu)$. Then we have

$$
\begin{aligned}
& \left|D_{z}^{-\delta} f(z)\right| \\
\geq & \left\{\frac{\Gamma(p+1)}{\Gamma(p+1+\delta)}-\frac{(p-\mu \lambda-\alpha) \Gamma(p+1) \Gamma(n+p+1-\lambda)}{[p+\mu(n-\lambda)] \Gamma(n+p+1+\delta) \Gamma(p+1-\lambda)}|z|^{n}\right\}|z|^{p+\delta}
\end{aligned}
$$

and

$$
\begin{aligned}
& \left|D_{z}^{-\delta} f(z)\right| \\
\leq & \left\{\frac{\Gamma(p+1)}{\Gamma(p+1+\delta)}+\frac{(p-\mu \lambda-\alpha) \Gamma(p+1) \Gamma(n+p+1-\lambda)}{[p+\mu(n-\lambda)] \Gamma(n+p+1+\delta) \Gamma(p+1-\lambda)}|z|^{n}\right\}|z|^{p+\delta}
\end{aligned}
$$

for $\delta>0$ and $z \in U$. The result is sharp. 
Proof. Let

$$
\begin{aligned}
F(z) & =\frac{\Gamma(p+1+\delta)}{\Gamma(p+1)} z^{-\delta} D_{z}^{-\delta} f(z) \\
& =z^{p}-\sum_{k=n+p}^{\infty} \frac{\Gamma(k+1) \Gamma(p+1+\delta)}{\Gamma(k+1+\delta) \Gamma(p+1)} a_{k} z^{k} \\
& =z^{p}-\sum_{k=n+p}^{\infty} D(k) a_{k} z^{k} \quad(z \in U)
\end{aligned}
$$

where

$$
D(k)=\frac{\Gamma(k+1) \Gamma(p+1+\delta)}{\Gamma(k+1+\delta) \Gamma(p+1)} \quad(k \geq n+p ; p, n \in \mathbb{N} ; \delta>0) .
$$

Since $D(k)$ is a decreaing function of $k$ when $\delta>0$, we get

$$
0<D(k) \leq D(n+p)=\frac{\Gamma(n+p+1) \Gamma(p+1+\delta)}{\Gamma(n+p+1+\delta) \Gamma(p+1)} .
$$

Thus, by using (5.6) and (5.15), we deduce that

$$
\begin{aligned}
& |F(z)| \geq|z|^{p}-D(n+p)|z|^{n+p} \sum_{k=n+p}^{\infty} a_{k} \\
& \quad \geq|z|^{p}-\frac{(p-\mu \lambda-\alpha) \Gamma(p+1+\delta) \Gamma(n+p+1-\lambda)}{[p+\mu(n-\lambda) \Gamma(n+p+1+\delta) \Gamma(p+1-\lambda)}|z|^{n+p} \quad(z \in U)
\end{aligned}
$$

and

$$
\begin{aligned}
& |F(z)| \leq|z|^{p}+D(n+p)|z|^{n+p} \sum_{k=n+p}^{\infty} a_{k} \\
& \quad \leq|z|^{p}+\frac{(p-\mu \lambda-\alpha) \Gamma(p+1+\delta) \Gamma(n+p+1-\lambda)}{[p+\mu(n-\lambda) \Gamma(n+p+1+\delta) \Gamma(p+1-\lambda)}|z|^{n+p} \quad(z \in U)
\end{aligned}
$$

which yield the inequalities (5.13) and (5.14) of Theorem 6 . The equalities in (5.13) and (5.14) are attained for the function $f(z)$ given by

$$
\begin{aligned}
D_{z}^{-\delta} f(z)= & \left\{\frac{\Gamma(p+1)}{\Gamma(p+1+\delta)}\right. \\
& \left.-\frac{(p-\mu \lambda-\alpha) \Gamma(p+1+\delta) \Gamma(n+p+1-\lambda)}{[p+\mu(n-\lambda)] \Gamma(n+p+1+\delta) \Gamma(p+1-\lambda)} z^{n}\right\} z^{p+\delta}
\end{aligned}
$$

or, equivalently, by the function $f(z)$ given by (3.2).

Putting $p=1$ in Theorem 6, we obtain the following result. 
Corollary 5. Let the function $f(z)$ defined by (1.1) (with $p=1$ ) be in the class $F_{\lambda}(n, \alpha, \mu)$. Then we have

$$
\begin{aligned}
& \left|D_{z}^{-\delta} f(z)\right| \\
& \quad \geq \frac{|z|^{1+\delta}}{\Gamma(2+\delta)}\left\{1-\frac{(1-\mu \lambda-\alpha) \Gamma(2+\delta) \Gamma(n+2-\lambda)}{[1+\mu(n-\lambda)] \Gamma(n+2+\delta) \Gamma(2-\lambda)}|z|^{n}\right\}
\end{aligned}
$$

and

$$
\begin{aligned}
& \left|D_{z}^{-\delta} f(z)\right| \\
& \quad \leq \frac{|z|^{1+\delta}}{\Gamma(2+\delta)}\left\{1+\frac{(1-\mu \lambda-\alpha) \Gamma(2+\delta) \Gamma(n+2-\lambda)}{[1+\mu(n-\lambda)] \Gamma(n+2+\delta) \Gamma(2-\lambda)}|z|^{n}\right\}
\end{aligned}
$$

for $\delta>0$ and $z \in U$. The result is sharp.

Remark 5. (i) We note that the result obtained by Altintas et al. [1, Theorem 3] is not correct. The correct result is given by Corollary 5;

(ii) Putting $\lambda=0$ and $n=1$ in Theorem 6 , we obtain the result obtained by Aouf and Darwish [2, Theorem 9].

Using arguments similar to those in the proof of Theorem 6, we obtain the following result.

Theorem 7. Let the function $f(z)$ defined by (1.1) be in the class $F_{\lambda}(n, p$, $\alpha, \mu)$. Then we have

$$
\begin{aligned}
& \left|D_{z}^{\delta} f(z)\right| \\
\geq & \left\{\frac{\Gamma(p+1)}{\Gamma(p+1-\delta)}-\frac{(p-\mu \lambda-\alpha) \Gamma(p+1) \Gamma(n+p+1-\lambda)}{[p+\mu(n-\lambda)] \Gamma(n+p+1-\delta) \Gamma(p+1-\lambda)}|z|^{n}\right\} z^{p-\delta}
\end{aligned}
$$

and

$$
\begin{aligned}
& \left|D_{z}^{\delta} f(z)\right| \\
\leq & \left\{\frac{\Gamma(p+1)}{\Gamma(p+1-\delta)}+\frac{(p-\mu \lambda-\alpha) \Gamma(p+1) \Gamma(n+p+1-\lambda)}{[p+\mu(n-\lambda)] \Gamma(n+p+1-\delta) \Gamma(p+1-\lambda)}|z|^{n}\right\} z^{p-\delta}
\end{aligned}
$$

for $0 \leq \delta<1$ and $z \in U$. The result is sharp.

Putting $p=1$ in Theorem 7 , we obtain the following result. 
Corollary 6. Let the function $f(z)$ defined by (1.1) (with $p=1$ ) be in the class $F_{\lambda}(n, \alpha, \mu)$. Then we have

$$
\left|D_{z}^{\delta} f(z)\right| \geq \frac{|z|^{1-\delta}}{\Gamma(2-\delta)}\left\{1-\frac{(1-\mu \lambda-\alpha) \Gamma(2+\delta) \Gamma(n+2-\lambda)}{[1+\mu(n-\lambda)] \Gamma(n+2-\delta) \Gamma(2-\lambda)}|z|^{n}\right\}
$$

and

$$
\left|D_{z}^{\delta} f(z)\right| \leq \frac{|z|^{1-\delta}}{\Gamma(2-\delta)}\left\{1+\frac{(1-\mu \lambda-\alpha) \Gamma(2+\delta) \Gamma(n+2-\lambda)}{[1+\mu(n-\lambda)] \Gamma(n+2-\delta) \Gamma(2-\lambda)}|z|^{n}\right\}
$$

for $0 \leq \delta<1$ and $z \in U$. The result is sharp.

Remark 6. (i) We note that the result obtained by Altintas et al. [1, Theorem 4] is not correct. The correct result is given by Corollary 6;

(ii) Putting $\lambda=0$ and $n=1$ in Theorem 7 , we obtain the result obtained by Aouf and Darwish [2, Theorem 10].

Acknowledgements. The authors are thankful to the referee for his comments and suggestions.

\section{REFERENCES}

[1] O. Altintas, H. Irmark and H. M. Srivastava, A subclass of analytic functions defined by using certain operators of fractional calculus, Comput. Math. Appl., 30 (1) (1995), $1-9$.

[2] M. K. Aouf and H. E. Darwish, Basic properties and characterizations of a certain class of analytic functions with negative coefficients, II, Utilitas Math., 46 (1994), $167-177$.

[3] S. D. Bernardi, Convex and starlike univalent functions, Trans. Amer. Math. Soc. 135(1969), 429-446.

[4] S. S. Bhoosnurmath and S. R. Swamy, Certain classes of analytic functions with negative coefficients, Indian J. Math., 27 (1985), 89-89.

[5] M. -P. Chen, H. Irmak and H. M. Srivastava, Some families of multivalently analytic functions with negative coefficients, J. Math. Anal. Appl., 214 (1997), 674-490.

[6] S. K. Lee, S. Owa and H. M. Srivastava, Basic properties and characterizations of a certain class of analytic functions with negative coefficients, Utilitas Math. 36 (1989), 121-128.

[7] R. J. Libera, Some classes of regular univalent functions, Proc. Amer. Math. Soc., 16 (1969), 755-758.

[8] A. E. Livingston, On the radius of univalence of cerain analytic functions, Proc. Amer. Math. Soc., 17 (1966), 352-357.

[9] S. Owa, On the distortion theorems, I, Kyungpook Math. J., 18 (1978), 55-59.

[10] H. M. Srivastava and M. K. Aouf, A certain fractional derivative operator and its applications to a new class of analytic and multivalent functions with negative coeffcients, I and II, J. Math. Anal. Appl., 171 (1992), 1-13; ibid. 192 (1995), 973-688.

[11] H. M. Srivastava and S. Owa (Editors), Univalent Functions, Fractional Calculus and Their Applivations, Halsted Press (Ellis Horwood Limited, Chichester), John Wiley and Sons, New York, Chichester, Brisbane and Toronto, 1989. 
[12] H. M. Srivastava and S. Owa (Editors), Current Topics in Analytic Function Theory, World Scientific Publishing Company, Singapore, New Jersey, London and Hong Kong, 1992.

(Received: September 3, 2011)

(Revised: January 17, 2012)
M. K. Aouf

Faculty of Science

Mansoura University

Mansoura 35516, Egypt

E-mail: mkaouf127@yahoo.com

B. A. Frasin

Department of Mathematics

Al- Al-Bayt University

P. O. Box: 130095 Mafraq

Jordan

E-mail: bafrasin@yahoo.com 